\title{
Non-visible Deformations
}

Jean-Denis DUROU ${ }^{1}$, Laurent MASCARILLA ${ }^{1}$, and Didier PIAU ${ }^{2}$

1 Université Paul Sabatier (Toulouse III) IRIT 118, route de Narbonne 31062

Toulouse Cedex, France tel: $(+33) 561.556 .882$; fax: $(+33) 561.556 .258$

E-mail: $\{$ durou,mascaril\}@irit.fr

${ }^{2}$ Université Claude Bernard (Lyon I) Laboratoire de Probabilités 43, boulevard du 11 Novembre 191869622 Villeurbanne Cedex, France tel: $(+33) 472.431 .260$; fax: (+33)472.431.266 E-mail: piau@jonas.univ-lyon1.fr

\begin{abstract}
The number of non isomorphic solutions of the eikonal equation can be infinite. We show that there can exist a whole family of non isomorphic solutions, indexed by a continuous parameter. This implies, first, that the general problem of shape from shading can be ill-posed when no additional condition on the shape is imposed. This is in contradiction with what is sometimes stated in the literature of shape from shading. Furthermore, this implies that there can exist non visible deformations of a given surface, i.e., continuous deformations of the surface which do not modify the image.
\end{abstract}

Keywords: shape from shading, eikonal equation, ill-posed problem, shape reconstruction.

\section{Introduction}

The determination of the shape of a surface from a single view of this surface can be achieved thanks to several criteria: texture, shadows, contours, shading. For a non textured surface, without edge nor hidden part, and if no silhouette is visible in the image, only the last of these criteria can function. This method is called shape from shading. B.K.P. Horn pioneered the work in this field in $1970[1,2]$ and showed that its basis equation was:

$$
R\left(f_{x}(x, y), f_{y}(x, y)\right)=E(x, y),
$$

in which the following notations have been chosen:

- Each point $(x, y)$ in the image is characterized by its shading $E(x, y)$;

- The shape of the viewed surface is defined by the equation $z=f(x, y)$, where the $z O z^{\prime}$ axis points towards the observer, and where $f(x, y)$ designates the height at point $(x, y)$;

- The behaviour of the surface, according to light re-emission, is described by the function $R\left(f_{x}, f_{y}\right)$, where $f_{x}$ and $f_{y}$ design the partial derivatives of $f$ in relation to $x$ and to $y$. 
For a surface lit from the direction of the observer, that is from the direction of the $z O z^{\prime}$ axis, it can easily be proved that $R\left(f_{x}, f_{y}\right)$ is a function of form $r\left(f_{x}{ }^{2}+f_{y}{ }^{2}\right)$, which means that the shading read in the image does not depend on anything else than the slope of the surface. In this case, equation (1) can be rewritten as:

$$
f_{x}(x, y)^{2}+f_{y}(x, y)^{2}=g(x, y),
$$

where $g(x, y)=r^{-1} \circ E(x, y)$ is known. This equation is called the eikonal equation. It is only a particular case of equation (1), but many authors have shown interest in its resolution [3-5]. It is a non linear partial derivatives equation of first order in $f(x, y)$, as well as (1). It is of major interest to state whether such an equation has a finite number of non isomorphic solutions (i.e., solutions which describe really different shapes) or not, in the absence of boundary conditions, that is if one does not a priori know the height at any point in the image.

In this paper, we concentrate on the surface $S_{1}$, defined by the equation $z=$ $2 x^{2}+y^{2}$. This surface is represented in figure 1 .

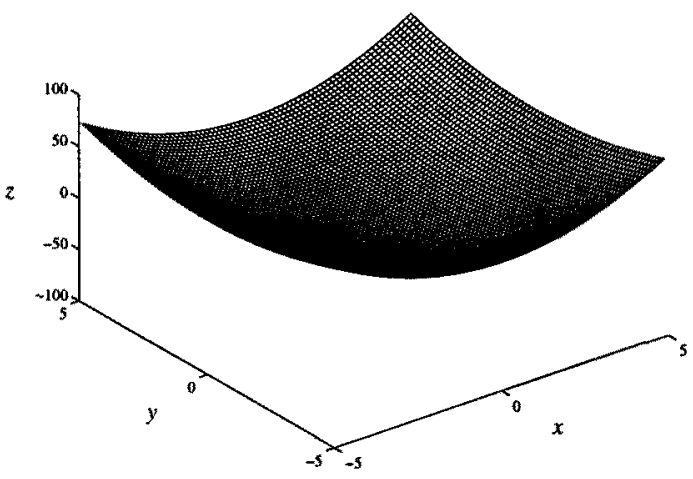

Fig. 1. Surface $z=2 x^{2}+y^{2}$.

Its image, computed with the Matlab 4.2 software, under the hypotheses of front lighting and of diffuse reflection, is represented in figure 2. 


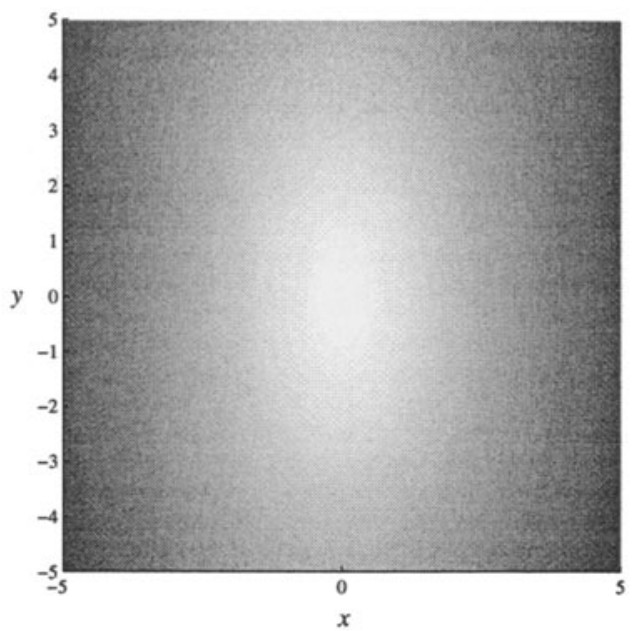

Fig. 2. Surface $z=2 x^{2}+y^{2}$ lit from the direction of the observer.

We would like to answer the following question: how many surfaces correspond to the same image as that of $S_{1}$, with front lighting? This can be reformulated as the determination of the number of solutions of the eikonal equation corresponding to the surface $S_{1}$, which is:

$$
f_{x}(x, y)^{2}+f_{y}(x, y)^{2}=16 x^{2}+4 y^{2} .
$$

It is obvious that if a surface $S$ defined by $z=f(x, y)$ is a solution of (3), then the surfaces defined by $z=f(x, y)+c$ and $z=-f(x, y)+c$ are solutions too, where $c$ designates any real value. These surfaces are what we call isomorphic to $S$ solutions. So, $S_{2}$ defined by $z=-\left(2 x^{2}+y^{2}\right)$ is an isomorphic to $S_{1}$ solution of (3). Besides $S_{1}$ and $S_{2}$, it is obvious that the surface $S_{3}$ defined by $z=2 x^{2}-y^{2}$ is a solution, as well as the isomorphic to $S_{3}$ surface $S_{4}$, defined by $z=-\left(2 x^{2}-y^{2}\right)$. We show that equation (3) has in fact many more analytical solutions than the four ones aforementioned. Actually, we prove the following theorem, which is the main result of this paper:

\section{Theorem}

There exists a family $\left(f_{c}\right)_{c \in \mathbb{R}_{+}}$of functions $f_{c}: \mathbb{R}^{2} \rightarrow \mathbb{R}$ verifying the following properties:

1. For $c>0, f_{c}$ is analytical and real on the disc $D(0, c)$;

2. For $c>0, f_{c}$ is a solution of (3) in the neighbourhood $D(0, c)$ of $(0,0)$;

3. The function $(c, x, y) \mapsto f_{c}(x, y)$ is continuous on $\mathbb{R}_{+} \times \mathbb{R}^{2}$; 


\section{If $c \neq c^{\prime}$, then $f_{c}$ and $f_{c^{\prime}}$ are not isomorphic.}

Each function $f_{c}, c>0$, corresponds thus to a surface, the image of which, when lit from the direction of the observer and restricted to the disc $D(0, c)=$ $\left\{(x, y) \in \mathbb{R}^{2} ; x^{2}+y^{2}<c^{2}\right\}$, coincides with the image of surface $S_{1}$ represented in figure 2. Therefore, it is possible to conceive, starting from one of the solutions $f_{c}, c>0$, a continuous deformation for which the image does not change in a neighbourhood of the origin. This explains the somewhat surprising title of our paper: non visible deformations.

How does this theorem take place in the field of shape from shading? Among the papers dealing with the resolution of the eikonal equation, those of Brooks, Chojnacki and Kozera [5] and of Horn, Szelisky and Yuille [6] are particularly interesting. Actually, it is proved, in these papers, that certain eikonal equations may admit no continuously differentiable solution, which is yet the largest class of solution, since an eikonal equation cannot admit any non-differentiable solution. We provide a result corresponding to the opposite situation, when proving the existence of an eikonal equation for which there exists an infinity of analytical solutions in a neighbourhood of the origin, which are not only smooth, but also the most regular solutions that can exist. These two opposite extreme situations consequently show that the number of solutions of the eikonal equation (2) may vary from zero to infinity, with respect to its second member $g(x, y)$. A certain number of authors have searched for characteristics concerning this second member $g(x, y)$, so that equation (2) admits only one continuously differentiable solution $[3,4,7]$. Let us mention that all these theorems of unicity suppose the existence of a silhouette in the analysed image, i.e., the existence of a closed curve, along which $g(x, y)$ is infinite (the image represented in figure 2 does not correspond to this situation). Such a situation is interesting, because it satisfies the initial wish of Horn [1], who wanted to compute, for any image, one surface exactly. Nevertheless, it is obvious that this situation can take place only in particular cases, since shape from shading is the inverse problem of image formation, and since the relation between all the possible surfaces and all the possible images is not a bijection. Here we prove that this inverse problem may be sometimes ill-posed, which means that there exists an infinity of solutions (this is in contradiction with what is claimed in [8]).

\section{A one parameter family of solutions of (3)}

In this section, we exhibit a family $\left(f_{c}\right)_{c \in \mathbb{R}_{+}}$of functions $f: \mathbb{R}^{2} \rightarrow \mathbb{R}$ satisfying properties 1-2-3-4 of the theorem. These functions verify also the two following properties:

5. The function $(c, x, y) \mapsto f_{c}(x, y)$ is real and analytical on $\left(\mathbb{R}_{+}^{*} \times \mathbb{R}^{2}\right) \backslash \Delta$, with $\Delta=\{y=0, x+c \leq 0\}$

6. - If c tends towards $+\infty$, then $f_{c}(x, y)$ tends towards $f_{\infty}(x, y)=2 x^{2}-y^{2}$;

- If $c$ tends towards $0^{+}$, then $f_{c}(x, y)$ tends towards $f_{0}(x, y)=2 x|x|+y^{2}$. 
In fact, for $c \neq 0, f_{c}$ is even analytical on $\mathbb{R}^{2} \backslash \Delta_{c}$, with $\left.\left.\Delta_{c}=\right]-\infty,-c\right] \times\{0\}$. At $P_{c}=(-c, 0), f_{c}$ is differentiable, but not of class $C^{2}$. At any point on $\Delta_{c}$ different to $P_{c}, f_{c}$ is continuous but not differentiable.

In this paper, we demonstrate only property 2 of the theorem, when proving that $f_{c}$ is a solution of $(3)$ on $\mathbb{R}^{2} \backslash \Delta_{c}$, as well as at point $P_{c}$.

Let $c \geq 0$. We define $f_{c}$ by the following:

$$
f_{c}(x, y)=2 x^{2}+y^{2}+\frac{16}{3} g_{c}(x, y)\left(x+c-g_{c}(x, y)\right),
$$

where the family of functions $\left(g_{c}\right)_{c \in \mathbb{R}_{+}}$is going to be defined.

Remark: The definition of $f_{c}(x, y)$, as well as a certain number of other definitions which are going to be given, may appear to be very arbitrary. Nevertheless, the whole reasoning which allows us to find the functions $f_{c}(x, y)$ is tedious, and we have decided not to show it in full in this paper.

Let $(x, y) \in \mathbb{R}^{2}$. Let us note:

$$
\left\{\begin{array}{l}
a=x+c, \\
b=\frac{27}{32} c y^{2}, \\
r=a^{3}+4 b, \\
s=8 b\left(a^{3}+2 b\right) .
\end{array}\right.
$$

Let us consider the following equation, where the unknown is $g$ :

$$
\Phi(g, a, b)=0,
$$

and where $\Phi(g, a, b)$ is defined by:

$$
\Phi(g, a, b)=g^{3}-\frac{3}{4} a^{2} g-\frac{1}{4}\left(a^{3}+4 b\right) .
$$

At any point $(x, y)$, the function $g_{c}(x, y)$ equals, by definition, the greatest real solution of equation (9). It can be shown [9] that the analytical expression of $g_{c}(x, y)$ is:

$$
g_{c}(x, y)=\frac{1}{2}(\sqrt[3]{r+\sqrt{s}}+\sqrt[3]{r-\sqrt{s}})
$$

where we designate by $\sqrt{s}$, when $s<0$, the value $i \sqrt{-s}$, where $i^{2}=-1$, and by $\sqrt[3]{ }$, when the argument is complex, the complex cubic root having the greatest real part. It is easy to show that expression (11) is always equal to or greater than zero.

In figure 3 , function $g_{1}(x, y)$ has been represented. In figure 4 , function $f_{1}(x, y)$, computed from $g_{1}(x, y)$ thanks to (4), has been represented. 


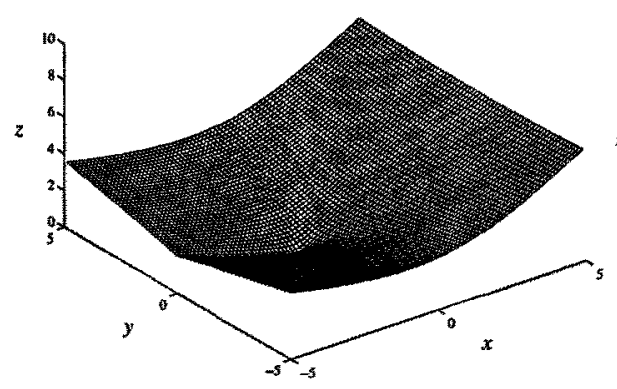

Fig.3. $g_{1}(x, y)$.

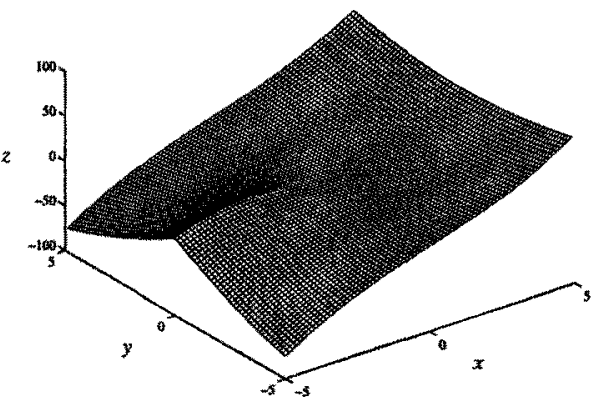

Fig.4. $f_{1}(x, y)$.

\section{$4 f_{c}(x, y)$ is a solution of equation (3)}

Let $c>0$. Let us compute the two partial derivatives $g_{x}$ and $g_{y}$ of $g_{c}(x, y)$ on $\mathbb{R}^{2} \backslash \Delta_{c}$. When deriving equation (9) in relation to $x$ and to $y$, and when using expression (10) of $\Phi(g, a, b)$, as well as expressions (5) and (6) of $a$ and of $b$, we obtain:

$$
\left\{\begin{array}{l}
\left(g^{2}-\frac{1}{4} a^{2}\right) g_{x}=\frac{1}{2} a g+\frac{1}{4} a^{2} \\
\left(g^{2}-\frac{1}{4} a^{2}\right) g_{y}=\frac{9}{16} c y .
\end{array}\right.
$$

It can be shown [9] that $2 g+a$ never equals zero. Equations (12) and (13) can then be rewritten:

$$
\left\{\begin{array}{l}
(a-2 g) g_{x}=-a \\
(a-2 g) g_{y}=-\frac{9}{4} \frac{c y}{2 g+a} .
\end{array}\right.
$$

The partial derivatives of $f_{c}(x, y)$, defined by (4), in relation to $x$ and to $y$, give on the other hand:

$$
\left\{\begin{array}{l}
f_{x}=4 x+\frac{16}{3} g+\frac{16}{3}(a-2 g) g_{x} \\
f_{y}=2 y+\frac{16}{3}(a-2 g) g_{y} .
\end{array}\right.
$$

When using (14) and (15), these expressions become:

$$
\left\{\begin{array}{l}
f_{x}=4 x+\frac{16}{3}(g-a) \\
f_{y}=2 y-12 \frac{c y}{2 g+a} .
\end{array}\right.
$$


The first member of equation (3) takes consequently the following form:

$$
f_{x}^{2}+f_{y}^{2}=16 x^{2}+4 y^{2}+A
$$

with:

$$
A=8 x \frac{16}{3}(g-a)+\left(\frac{16}{3}\right)^{2}(g-a)^{2}-48 \frac{c y^{2}}{2 g+a}+144 \frac{c^{2} y^{2}}{(2 g+a)^{2}} .
$$

Now, when using (6) and factorizing the expression of $\Phi(g, a, b)$ given in (10), equation (9) takes the form:

$$
12 \frac{c y^{2}}{(2 g+a)^{2}}=\frac{32}{9}(g-a),
$$

which gives, when reporting in (21):

$$
A=\frac{64}{9}(g-a)(6 x+4(g-a)-2(2 g+a)+6 c) .
$$

The last factor in equation 23 equals zero, thus $f_{c}(x, y)$ is a solution of equation (3) on $\mathbb{R}^{2} \backslash \Delta_{c}$. It is easy to verify that it also verifies (3) at point $P_{c}$, then finally $f_{c}(x, y)$ is a solution of equation (3) on $\left.\mathbb{R}^{2} \backslash\right]-\infty,-c[\times\{0\}$.

In figure 5, we represented the image of the surface $z=f_{2}(x, y)$, with front lighting.

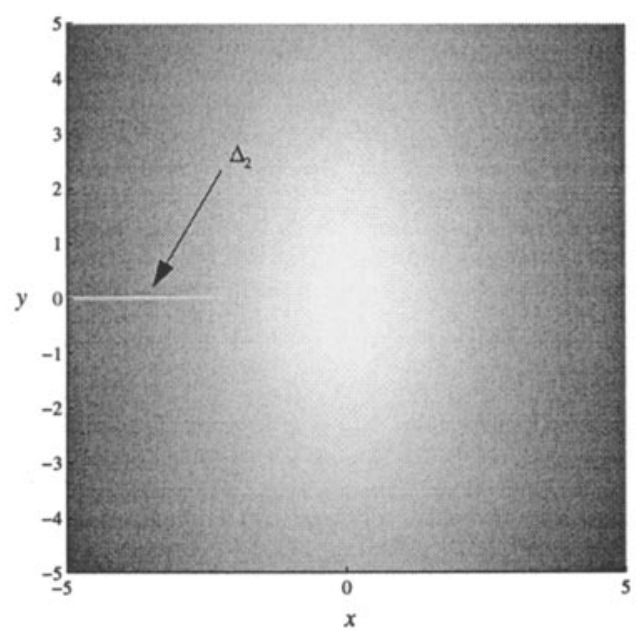

Fig. 5. Surface $z=f_{2}(x, y)$ lit from the direction of the observer. 
This image is actually identical to that represented in figure 2, except on $\Delta_{2} \backslash$ $\left\{P_{2}\right\}$, where $f_{2}(x, y)$ is not derivable.

As for $f_{0}(x, y)$, it can be verified without any difficulty that it is a solution of (3) on $\mathbb{R}^{2}$, knowing that:

$$
f_{0}(x, y)=2 x|x|+y^{2}
$$

\section{Application}

A surprising application of the theorem we just proved is the existence of non visible deformations. Such a deformation was simulated thanks to the Matlab 4.2 software. The image remains actually unchanged. On the other hand, if we modify, even slightly, the observation direction or the lighting direction, the deformation becomes visible. This means that such deformations are non visible within the context of monovision, but not within the context of stereovision. This situation shows, when necessary, the superiority of the stereovision methods over the monovision methods, with regard to shape reconstruction.

\section{References}

1. Horn B.K.P. "Shape from Shading: a Method for Obtaining the Shape of a Smooth Opaque Object from One View". PhD thesis, Department of Electrical Engineering, MIT, 1970.

2. Horn B.K.P. "Obtaining Shape from Shading Information". In The Psychology of Computer Vision, chapter 4, pages 115-155. P.H. Winston (ed.), New York, 1975.

3. Bruss A.R. "The Eikonal Equation: Some Results Applicable to Computer Vision". Journal of Mathematical Physics, 23(5):890-896, 1982.

4. Oliensis J. "Uniqueness in Shape from Shading". International Journal of Computer Vision, 6(2):75-104, 1991.

5. Brooks M.J., Chojnacki W., and Kozera R. "Shading without Shape". Quarterly of Applied Mathematics, L(1):27-38, 1992.

6. Horn B.K.P., Szeliski R.S., and Yuille A.L. "Impossible Shaded Images". IEEE PAMI, 15(2):166-169, 1993.

7. Blake A., Zisserman A., and Knowles G. "Surface Descriptions from Stereo and Shading". Image and Vision Computing, 3(4):183-191, 1985.

8. Durou J.D. "Reconnaissance du relief à partir de l'éclairement". PhD thesis, Université Paris XI-Orsay, 1993.

9. Durou J.D., Mascarilla L., and Piau D. "Non visible distortions". Submitted to IEEE PAMI. 